\title{
RECTIFIABLE DIAMETERS OF THE GRASSMANN SPACES OF CERTAIN VON NEUMANN ALGEBRAS AND C*-ALGEBRAS
}

\section{SHUANG ZHANG}

\begin{abstract}
We prove that any two homotopic projections in certain $\mathrm{C}^{*}$-algebras can be connected by a rectifiable path of projections whose length is bounded by a universal constant. In comparison, N.C. Phillips (1992) proved that there are $\mathrm{C}^{*}$ algebras in which such a universal constant does not exist. Our techniques are to estimate the number of symmetries needed to conjugate any two homotopic projections and to factor a unitary in the identity path component as a product of a limited number of symmetries.
\end{abstract}

\section{Introduction.}

Let $\mathcal{A}$ be a $\mathrm{C}^{*}$-algebra and $\mathcal{P}(\mathcal{A})$ denote the space of all nontrivial projections $(\neq 0,1)$ in $\mathcal{A}$ equipped with the norm topology, called the Grassmann space of $\mathcal{A}$. Investigations of $\mathcal{P}(\mathcal{A})$ from various angles have been made during the last several decades by M. Breuer, L.G. Brown, G. Corach, J. Dixmier, H. Porta, L. Recht, N. Salinas, and D.R. Wilkins, among others (see the references of $[\mathbf{B r}]$ ). For von Neumann algebras and certain simple $\mathrm{C}^{*}$-algebras the homotopy groups of $\mathcal{P}(\mathcal{A})$ are closely related to the K-theory of $\mathcal{A}$ [Bre1, Bre2, Zh11, Zh5]. L.G. Brown has recently considered a rectifiable metric defined on $\mathcal{P}(\mathcal{L}(\mathcal{H}))$ by

$$
d_{r}(p, q):=\inf \{\text { length of } \gamma: \gamma \text { is a rectifiable path joining } p \text { and } q\},
$$

where $\mathcal{L}(\mathcal{H})$ is the algebra of all bounded operators on a separable Hilbert space $\mathcal{H}$. He proved that $d_{r}(p, q)=\sin ^{-1}\|p-q\|$ if $\|p-q\|<1$ and the range of $d_{r}(p, q)$ is the whole interval $[0, \pi]$. He also proved that a minimizing path connecting $p$ and $q$ exists if and only if $\operatorname{rank}(p \wedge(1-q))=\operatorname{rank}(q \wedge(1-p))$ $([\mathrm{Br}, 2.12])$.

The main purpose of this article is to estimate the number of symmetries (self-adjoint unitaries) that conjugate two homotopic projections and the lengths of rectifiable paths which join two homotopic projections in certain $\mathrm{C}^{*}$-algebras. We will be concerned with the following invariant

$$
\sup _{p, q}\left\{d_{r}(p, q): p \approx q \text { in } \mathcal{P}(\mathcal{A})\right\}
$$


where ' $p \approx q$ ' means that two projections $p$ and $q$ are in the same path component of $\mathcal{P}(\mathcal{A})$. This invariant measures the uniform upper bound of the infimum of the lengths of rectifiable paths connecting homotopic projections in $\mathcal{P}(\mathcal{A})$, and hence, can be regarded as a rectifiable diameter of $\mathcal{P}(\mathcal{A})$. N.C. Phillips has later investigated the number $(*)$ further in $[\mathbf{P h 1}]$ where the invariant $(*)$ is called the $C^{*}$-projective length of $\mathcal{A}$.

First, we consider $\mathrm{C}^{*}$-algebras of real rank zero, including all von $\mathrm{Neu}$ mann factors. It turns out that any two homotopic projections in a type $\mathrm{II}_{1}$ factor, in a type III factor, in a type $\mathrm{II}_{\infty}$ factor, and in $\mathcal{L}(\mathcal{H})$ can be conjugated by 2 (or $1+\epsilon$ ), 2,3 ( or $2+\epsilon$ ), and 3 (or $2+\epsilon$ ) symmetries, respectively, where by $n+\epsilon$ symmetries we mean 'by a product of $n$ symmetries and a unitary which is arbitrarily close to the identity in norm'. As a consequence, we recapture the following results of $\mathrm{L}$. G. Brown in $[\mathrm{Br}, 2.12,3.3]$ : The $\mathrm{C}^{*}$-projective length $(*)$ is less than or equal to

$$
\begin{cases}\frac{\pi}{2} & \text { if } \mathcal{A} \text { is a factor of type } \mathrm{II}_{1} ; \\ \pi & \text { if } \mathcal{A} \text { is a factor of type } \mathrm{III}, \text { or } \mathrm{II}_{\infty}, \text { or } \mathcal{L}(\mathcal{H}) .\end{cases}
$$

If $\mathcal{A}$ is a $\mathrm{C}^{*}$-algebra of real rank zero with cancellation, then any two homotopic projections in $\mathcal{A}$ can be conjugated by 2 (or $1+\epsilon$ ) symmetries; if $\mathcal{A}$ is purely infinite and simple, then any two homotopic projections in $\mathcal{A}$ can be conjugated by 2 symmetries. As a consequence, the $\mathrm{C}^{*}$-projective length $(*)$ is less than or equal to

$$
\begin{cases}\frac{\pi}{2} & \text { if } \mathcal{A} \text { has cancellation (i.e., } \operatorname{tsr}(\mathcal{A})=1) \\ \pi & \text { if } \mathcal{A} \text { is purely infinite and } \operatorname{simple} .\end{cases}
$$

Secondly, we consider $\mathrm{C}^{*}$-algebras with one of the following forms

$$
\mathcal{A} \otimes \mathcal{K}, \quad \mathcal{B} \otimes \mathcal{L}\left(\mathcal{H}_{\mathcal{C}}\right) \quad \text { and } \quad C(X, \tilde{\mathcal{D}}),
$$

where $\mathcal{B}$ is a unital $\mathrm{C}^{*}$-algebra, $\mathcal{C}$ is a $\sigma$-unital $\mathrm{C}^{*}$-algebra, $\mathcal{D}$ is a purely infinite, simple $\mathrm{C}^{*}$-algebra, $X$ is a compact Hausdorff space, and $\mathcal{L}\left(\mathcal{H}_{\mathcal{C}}\right)$ is isomorphic to the multiplier algebra $M(\mathcal{C} \otimes \mathcal{K})$ of $\mathcal{C} \otimes \mathcal{K}$. We prove that any unitary in $\mathcal{B} \otimes \mathcal{L}\left(\mathcal{H}_{\mathcal{C}}\right)$ can be written as a product of seven symmetries, and every unitary in the identity path component of the unitary group of $C(X, \tilde{\mathcal{D}})$ can be approximated by a product of six symmetries. Consequently, the $\mathrm{C}^{*}$-projective length $(*)$ of $\mathcal{A}$ is less than or equal to

$$
\begin{cases}\pi & \text { if } \mathcal{A} \cong \mathcal{A} \otimes \mathcal{K} \\ 3 \pi & \text { if } \mathcal{A} \text { has the form } \mathcal{B} \otimes \mathcal{L}\left(\mathcal{H}_{\mathcal{C}}\right) \\ 3 \pi & \text { if } \mathcal{A} \text { has the form } C(X, \tilde{\mathcal{D}})\end{cases}
$$


In particular, if the $\mathrm{C}^{*}$-algebra $\mathcal{B}$ above is the algebra of all complex numbers, then

$$
\mathcal{B} \otimes \mathcal{L}\left(\mathcal{H}_{\mathcal{C}}\right) \cong \mathcal{L}\left(\mathcal{H}_{\mathcal{C}}\right) \cong M(\mathcal{C} \otimes \mathcal{K})
$$

As a consequence, the $\mathrm{C}^{*}$-projective length $(*)$ of the multiplier algebra of any $\sigma$-unital stable $\mathrm{C}^{*}$-algebra is bounded above by $3 \pi$. If $\mathcal{C}$ is either a $\sigma$-unital infinite simple $\mathrm{C}^{*}$-algebra or $\mathcal{C}$ is a $\sigma$-unital $\mathrm{C}^{*}$-algebra with cancellation, then the upper bounds (III) above can be improved from $3 \pi$ to $2 \pi$ with the aid of [Zh8]. If $\mathcal{C}$ is a $\mathrm{C}^{*}$-algebra such that $R R\left(\mathcal{L}\left(\mathcal{H}_{\mathcal{C}}\right)\right)=0$, then the upper bounds (III) above can be improved from $3 \pi$ to $\pi$. These improvements can be done due to the fact that fewer symmetries are necessary to conjugate two homotopic projections.

In addition, by factoring each unitary in $\mathcal{B} \otimes \mathcal{L}\left(\mathcal{H}_{\mathcal{C}}\right)$ into a product of seven symmetries we show that $\mathcal{B} \otimes \mathcal{L}\left(\mathcal{H}_{\mathcal{C}}\right)$ is generated algebraically by projections, although $\mathcal{B}$ and $\mathcal{C}$ may contain no non-trivial projections at all. In particular, if either $\mathcal{B}=C(X)$ or $\mathcal{B}$ is the algebra of all complex numbers, then the multiplier algebra $M(\mathcal{C} \otimes \mathcal{K})$ and $C\left(X, \mathcal{L}\left(\mathcal{H}_{\mathcal{C}}\right)\right)$ are generated algebraically by projections. This includes the particularly important case $C(X, \mathcal{L}(\mathcal{H}))$. A similar conclusion holds in $C(X, \tilde{\mathcal{D}})$.

All proofs are carried out by elementary, but technical, constructions. In comparison with our results, N.C. Phillips very recently proved [Ph1] that the $\mathrm{C}^{*}$-projective length $(*)$ can be equal to $+\infty$ for some $\mathrm{C}^{*}$-algebras with the form $M_{2}(C(X))$ where $X$ is certain topological space. The reader is referred to $[\mathbf{P h} \mathbf{1}]$ for further developments on the $\mathrm{C}^{*}$-projective length $(*)$.

Acknowledgment. The author wishes to thank L.G. Brown and N.C. Phillips for their valuable comments on the preliminary version of this article.

\section{Preliminaries.}

Let $\tilde{\mathcal{A}}$ be the unital $\mathrm{C}^{*}$-algebra after joining an identity to $\mathcal{A}$ if $\mathcal{A}$ is nonunital, and let $\tilde{\mathcal{A}}=\mathcal{A}$ if $\mathcal{A}$ is unital. Two projections $p$ and $q$ in $\mathcal{P}(\mathcal{A})$ are said to be equivalent, denoted by $p \sim q$, if there exists a partial isometry $v$ in $\mathcal{A}$ such that $v v^{*}=p$ and $v^{*} v=q$. The equivalence class containing $p$ is denoted by $[p]$. Write $[p] \leq[q]$ if $p$ is equivalent to a subprojection of $q$. Two projections $p$ and $q$ are said to be unitarily equivalent, if there exists a unitary $u$ of $\tilde{\mathcal{A}}$ such that $u p u^{*}=q ; p$ and $q$ are said to be homotopic, denoted by $p \approx q$, if $p$ and $q$ are in the same path component of $\mathcal{P}(\mathcal{A})$. Of course, two homotopic projections are unitarily equivalent, and two unitarily equivalent projections are equivalent. But the converses do not hold in general.

Lemma 1. Assume that $\mathcal{A}$ is a $C^{*}$-algebra, $p, q \in \mathcal{P}(\mathcal{A})$, and $0<\delta<1$. Then $\|p-p q p\| \leq \delta^{2}$ and $\|(1-p) q(1-p)\| \leq \delta^{2}$ if and only if $\|p-q\| \leq \delta$. 
The equivalence still holds if all inequalities ' $\leq$ ' in the statement are replaced by ' $<$ '.

Proof. It is well known that $\|p-q\|=\max \{\|p(1-q)\|,\|q(1-p)\|\}$. In fact, one inequality $\geq$ follows from $(p-q)(1-p)=-q(1-p)$ and $(p-q)(1-q)=p(1-q)$, and the other $\leq$ follows from the following elementary estimate:

$$
\begin{aligned}
\|(p-q) \xi\| & =\|(p-q) p \xi+(p-q)(1-p) \xi\| \\
& =\|(1-q) p \xi-q(1-p) \xi\| \\
& \leq \max \{\|(1-q) p \xi\|,\|q(1-p) \xi\|\}
\end{aligned}
$$

where $\xi$ is any unit vector in a Hilbert space on which $\mathcal{A}$ is faithfully represented. Then both directions of the lemma follow immediately from the following equalities:

$$
\begin{aligned}
\|p(1-q)\| & =\|p-p q p\|^{\frac{1}{2}} \\
\|q(1-p)\| & =\|(1-p) q(1-p)\|^{\frac{1}{2}} .
\end{aligned}
$$

Lemma 2. Assume that $\mathcal{A}$ is a $C^{*}$-algebra, and $p, q \in \mathcal{P}(\mathcal{A})$. If $\|p-q\|<1$, then there is a symmetry $s$ in $C^{*}(p, q, 1)$ such that sps $=q$, where $C^{*}(p, q, 1)$ is the $C^{*}$-subalgebra of $\tilde{\mathcal{A}}$ generated by $p, q$ and the identity 1 .

Proof. This is also a well known fact. In fact, set $s:=\left(1-(p-q)^{2}\right)^{-\frac{1}{2}}(p+q-1)$. Then one can easily show that $s$ is a symmetry such that $s p s=q$.

From now on, we will identify $\mathcal{A}$ with its matrix decomposition according to the following decomposition of the identity

$$
\bigoplus_{i=1}^{n} p_{i}=1, \quad p_{i} \in P(\mathcal{A}), \quad p_{i} p_{j}=0 \quad(i \neq j) .
$$

This identification is a ${ }^{*}$-isomorphism which assigns to each $x$ in $\mathcal{A}$ the following matrix:

$$
\left(\begin{array}{cccc}
p_{1} x p_{1} & p_{1} x p_{2} & \ldots & p_{1} x p_{n} \\
p_{2} x p_{1} & \ddots & \ddots & \vdots \\
\vdots & \ddots & p_{n-1} x p_{n-1} & \vdots \\
p_{n} x p_{1} & \ldots & p_{n} x p_{n-1} & p_{n} x p_{n}
\end{array}\right)
$$

where the multiplication, addition, scalar multiplication, and the involution * follow the standard rules of matrix manipulations. The matrix above is called the matrix form of $x$ with respect to the decomposition $\bigoplus_{i=1}^{n} p_{i}=1$. 
Proposition 3. Suppose that $\mathcal{A}$ is a $C^{*}$-algebra.

(i) Let $p, q \in \mathcal{P}(\mathcal{A})$. Write the matrix form of $q$ with respect to $p \oplus(1-p)=$ 1 as follows:

$$
q=\left(\begin{array}{cc}
a & b \\
b^{*} & c
\end{array}\right)
$$

If $\max \{\|p-a\|,\|c\|\}<1$, then there exists a symmetry $s \in C^{*}(p, q, 1)$ such that $s q s=p$.

(ii) If $p_{0}$ and $p_{1}$ are two homotopic projections in $\mathcal{P}(\mathcal{A})$, then there are symmetries $s_{1}, s_{2}, \ldots, s_{l}$ of $\tilde{\mathcal{A}}$ such that $s_{1} s_{2} \ldots s_{l} p_{1} s_{l} \ldots s_{2} s_{1}=p_{0}$.

Proof. (i) Since $p-p q p=p-a$ and $(1-p) q(1-p)=c$, the conclusion (i) follows immediately from Lemma 1 and Lemma 2 .

(ii) Let $\{p(t): t \in[0,1]\}$ be a path in $\mathcal{P}(\mathcal{A})$ such that $p(0)=p_{0}$ and $p(1)=p_{1}$. Take a subdivision of $[0,1]$, say $0=t_{0}<t_{1}<t_{2}<\ldots<t_{l-1}<$ $t_{l}=1$, such that

$$
\max _{1 \leq i \leq l}\left\|p\left(t_{i}\right)-p\left(t_{i-1}\right)\right\|<1
$$

Set

$$
s_{i}=\left(1-\left(p\left(t_{i}\right)-p\left(t_{i-1}\right)\right)^{2}\right)^{-\frac{1}{2}}\left(p\left(t_{i}\right)+p\left(t_{i-1}\right)-1\right) \quad(1 \leq i \leq l) .
$$

Then $s_{1} s_{2} \ldots s_{l} p_{1} s_{l} \ldots s_{2} s_{1}=p_{0}$.

\section{Main Results.}

The main results of this article are labeled as Theorem 5, Theorem 9, and Theorem 11. These results will be used to study factorizations in the unitary group of certain $\mathrm{C}^{*}$-algebras [Zh9].

A $\mathrm{C}^{*}$-algebra $\mathcal{A}$ has real rank zero, denoted by $R R(\mathcal{A})=0$, if and only if every self-adjoint element of $\mathcal{A}$ can be approximated in norm by selfadjoint elements with finite spectrum $[\mathrm{BP}, 2.6]$. A simple $\mathrm{C}^{*}$-algebra $\mathcal{A}$ is purely infinite if and only if $R R(\mathcal{A})=0$ and every nonzero projection of $\mathcal{A}$ is infinite [Zh7, Part I, 1.2(ii)]. In a purely infinite, simple $\mathrm{C}^{*}$-algebra $\mathcal{A}$, two nontrivial projections $p$ and $q$ are equivalent if and only if $p$ and $q$ are homotopic $([\mathbf{E K}, 2.4]$ and [Zh7, Part II, 1.1]). If $R R(\mathcal{A})=0$ and $\mathcal{A}$ has cancellation, then two equivalent projections are necessarily homotopic $([\mathbf{Z h} 3,3.4])$. Here a $\mathrm{C}^{*}$-algebra is said to have cancellation, if the conditions $p_{1} \oplus r_{1} \sim p_{2} \oplus r_{2}$ and $r_{1} \sim r_{2}$ imply $p_{1} \sim p_{2}$, where $p_{1}, p_{2}, r_{1}, r_{2} \in \mathcal{P}(\mathcal{A})$. Under the condition $R R(\mathcal{A})=0, \mathcal{A}$ has cancellation if and only if $\operatorname{tsr}(\mathcal{A})=1$ $([\mathrm{Bl1}, 6.5])$.

The following is a key lemma which leads to Theorem 5 . 
Lemma 4. Assume that $A$ is a $C^{*}$-algebra with $R R(\mathcal{A})=0$ and $p, q \in$ $\mathcal{P}(\mathcal{A})$. Then the following hold:

(i) There exists a symmetry $s \in \tilde{\mathcal{A}}$ such that sps $=p_{1} \oplus p_{2}$, where $p_{1}$ is a projection in $q \mathcal{A} q$ and $p_{2}$ is a projection in $(1-q) \mathcal{A}(1-q)$.

(ii) If, in addition, $[q] \leq[1-q]$, then there exist two symmetries $s$ and $s^{\prime}$ in $\tilde{\mathcal{A}}$ such that $s^{\prime}$ spss $s^{\prime}=p_{1}^{\prime} \oplus p_{2}^{\prime}$, where $p_{1}^{\prime}$ and $p_{2}^{\prime}$ are two projections such that $p_{1}^{\prime} \leq q, p_{2}^{\prime} \leq 1-q$, and $\left[p_{1}^{\prime}\right] \leq\left[p_{2}^{\prime}\right]$.

Proof. (i) Write the matrix form of $p$ with respect to $q \oplus(1-q)=1$ as follows:

$$
p=\left(\begin{array}{cc}
q p q & q p(1-q) \\
(1-q) p q & (1-q) p(1-q)
\end{array}\right) .
$$

Using the construction in [Zh3, 2.1] (initiated by L.G. Brown for AF algebras and further developed in $[\mathbf{Z h 3}, 2.1]$ for $\mathrm{C}^{*}$-algebras of real rank zero), one can find for each positive number $\delta<1-\frac{1}{\sqrt{2}}$ a projection $p_{0}$ such that

$$
\left\|p-p_{0}\right\|<\delta
$$

where the matrix form of $p_{0}$ with respect to the decomposition $f_{0} \oplus(q-$ $\left.f_{0}\right) \oplus\left(1-q-e_{0}\right) \oplus e_{0}=1$ is

$$
p_{0}=\left(\begin{array}{cccc}
f_{0} & 0 & 0 & 0 \\
0 & a & b & 0 \\
0 & b^{*} & c & 0 \\
0 & 0 & 0 & e_{0}
\end{array}\right)
$$

Let us explain the construction of $a, b, c, f_{0}$, and $e_{0}$. The element $f_{0}$ above is a projection in $q \mathcal{A} q, e_{0}$ is a projection in $(1-q) \mathcal{A}(1-q), a:=$ $\left(q-f_{0}\right) p_{0}\left(q-f_{0}\right)$ is a positive element such that its spectrum $\sigma(a)$ is a finite subset of $[0,1)$, say $\sigma(a) \backslash\{0\}=\left\{t_{i}\right\}_{i=1}^{m}, b:=\left(q-f_{0}\right) p_{0}\left(1-q-e_{0}\right)$, and $c:=\left(1-q-e_{0}\right) p_{0}\left(1-q-e_{0}\right)$ is also a positive element in $\left(1-q-e_{0}\right) \mathcal{A}\left(1-q-e_{0}\right)$ such that $\sigma(c) \backslash\{0\}=\left\{1-t_{i}\right\}_{i=1}^{m} \subset[0,1)$. Furthermore, there is a close relation between $a$ and $c$ as follows. Write

$$
c=\sum_{i=1}^{m}\left(1-t_{i}\right) r_{i}
$$

where $\left\{r_{i}\right\}_{i=1}^{m}$ is a set of mutually orthogonal subprojections of $1-q-e_{0}$. Then

$$
a=v\left(\sum_{i=1}^{m} r_{i}-c\right) v^{*}=\sum_{i=1}^{m} t_{i} v r_{i} v^{*},
$$


where $v$ is some partial isometry in $\mathcal{A}$ such that $v^{*} v=\sum_{i=1}^{m} r_{i}$. Set $e:=v^{*} v$. The element $b$ is defined by

$$
b=v(e-c)^{\frac{1}{2}} .
$$

Set $v v^{*}:=f$. Then $f$ is a subprojection of $q-f_{0}$. Of course, $e f=0$, and $f$, $e$ are the range projections of $a$ and $c$, respectively. For more details of the construction of $p_{0}$ the reader is referred to [Zh3, 2.1].

Set

$$
r_{0}=\left(\begin{array}{cc}
v\left(\sum_{\left\{i: t_{i} \geq \frac{1}{2}\right\}} r_{i}\right. \\
0 & \sum_{\left\{i: t_{i}<\frac{1}{2}\right\}} r_{i}
\end{array}\right) .
$$

Then $r_{0}$ is a projection in $(e \oplus f) \mathcal{A}(e \oplus f)$. It follows from a simple calculation that

$$
v\left(\sum_{\left\{i: t_{i} \geq \frac{1}{2}\right\}} r_{i}\right) v^{*} b\left(\sum_{\left\{i: t_{i}<\frac{1}{2}\right\}} r_{i}\right)=0 .
$$

Consequently,

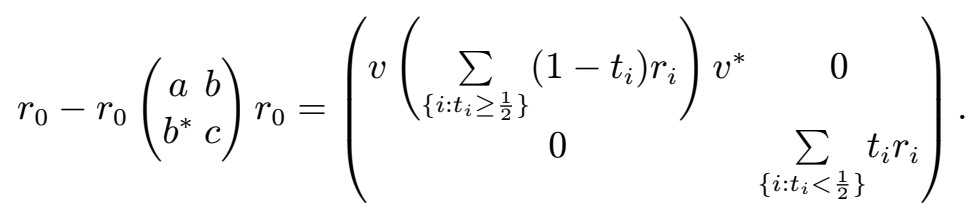

It follows that

$$
\left\|r_{0}-r_{0}\left(\begin{array}{cc}
a & b \\
b^{*} & c
\end{array}\right) r_{0}\right\| \leq \frac{1}{2} .
$$

Similarly, one can show

$$
\left\|\left(e \oplus f-r_{0}\right)\left(\begin{array}{cc}
a & b \\
b^{*} & c
\end{array}\right)\left(e \oplus f-r_{0}\right)\right\| \leq \frac{1}{2} .
$$

Applying Lemma 1, one has

$$
\left\|r_{0}-\left(\begin{array}{cc}
a & b \\
b^{*} & c
\end{array}\right)\right\| \leq \frac{1}{\sqrt{2}} .
$$

Set

$$
p_{0}^{\prime}=\left(\begin{array}{ccccc}
f_{0} & 0 & 0 & 0 \\
0 & v\left(\sum_{\left\{i: t_{i} \geq \frac{1}{2}\right\}} r_{i}\right) v^{*} & 0 & 0 \\
0 & 0 & \sum_{\left\{i: t_{i}<\frac{1}{2}\right\}} r_{i} & 0 \\
0 & 0 & 0 & e_{0}
\end{array}\right) .
$$


Then

$$
\left\|p_{0}-p_{0}^{\prime}\right\| \leq\left\|r_{0}-\left(\begin{array}{cc}
a & b \\
b^{*} & c
\end{array}\right)\right\| \leq \frac{1}{\sqrt{2}}
$$

It follows that

$$
\left\|p-p_{0}^{\prime}\right\| \leq\left\|p-p_{0}\right\|+\left\|p_{0}-p_{0}^{\prime}\right\| \leq \frac{1}{\sqrt{2}}+\delta<1 .
$$

Set

$$
p_{1}=f_{0} \oplus v\left(\sum_{\left\{i: t_{i} \geq \frac{1}{2}\right\}} r_{i}\right) v^{*} \text { and } p_{2}=e_{0} \oplus \sum_{\left\{i: t_{i}<\frac{1}{2}\right\}} r_{i} .
$$

Then $p_{0}^{\prime}=p_{1} \oplus p_{2}$, where $p_{1}$ is a projection in $q \mathcal{A} q$ and $p_{2}$ is a projection in $(1-q) \mathcal{A}(1-q)$. Applying Lemma 2 , one gets a symmetry $s$ in $\tilde{\mathcal{A}}$ such that sps $=p_{1} \oplus p_{2}$.

(ii) Let $s, p_{1}$, and $p_{2}$ be as in (i). Then $p_{1} \leq q$ and $[q] \leq[1-q]=$ $\left[p_{2}\right]+\left[1-q-p_{2}\right]$. By the Riesz decomposition property in $[\mathbf{Z h} \mathbf{6}, 1]$ and $[\mathrm{Zh} 2,2.2]$ one can write

$$
p_{1}=q_{1} \oplus q_{2},
$$

where $\left[q_{1}\right] \leq\left[p_{2}\right]$ and $\left[q_{2}\right] \leq\left[1-q-p_{2}\right]$. Take a partial isometry $w$ in $\mathcal{A}$ such that $w w^{*}=q_{2}$ and $w^{*} w \leq 1-q-p_{2}$. Set

$$
s_{1}^{\prime}:=w+w^{*}+\left(q_{2} \oplus\left(1-q-p_{2}\right)-w^{*} w\right) .
$$

Then $s_{1}^{\prime}$ is a symmetry in $\left(q_{2} \oplus\left(1-q-p_{2}\right)\right) \tilde{\mathcal{A}}\left(q_{2} \oplus\left(1-q-p_{2}\right)\right)$ such that $s_{1}^{\prime} q_{2} s_{1}^{\prime}=w^{*} w \leq 1-q-p_{2}$. Set

$$
p_{1}^{\prime}=q_{1}, \quad p_{2}^{\prime}=p_{2} \oplus s_{1}^{\prime} q_{2} s_{1}^{\prime}, \quad \text { and } \quad s^{\prime}=\left(q-q_{2}\right) \oplus p_{2} \oplus s_{1}^{\prime} .
$$

Then $s^{\prime}$ is a symmetry of $\tilde{\mathcal{A}}$ such that

$$
s^{\prime} \text { sps }^{\prime}=p_{1}^{\prime} \oplus p_{2}^{\prime}, \quad \text { and } \quad\left[p_{1}^{\prime}\right] \leq\left[p_{2}^{\prime}\right] .
$$

The proof is complete.

Theorem 5. Assume that $\mathcal{A}$ is a $C^{*}$-algebra with $R R(\mathcal{A})=0$ and $p, q$ $\in \mathcal{P}(\mathcal{A})$.

(i) If $\mathcal{A}$ has cancellation and two projections $p, q \in \mathcal{P}(\mathcal{A})$ are equivalent, then there exist for each positive number $\epsilon$ a symmetry $s$ and a unitary $u_{\epsilon}$ in $\tilde{\mathcal{A}}$ such that $\left\|u_{\epsilon}-1\right\|<\epsilon$ and $s u_{\epsilon} p u_{\epsilon}^{*} s=q$, and there exist two symmetries $s$ and $s^{\prime}$ in $\tilde{\mathcal{A}}$ such that $s^{\prime} p s^{\prime} s=q$. 
(ii) If $\mathcal{A}$ is a direct sum of finite dimensional $C^{*}$-algebras and finitely many copies of the algebra $\mathcal{K}$ of all compact operators, then two equivalent projections $p, q \in \mathcal{P}(\mathcal{A})$ can be conjugated by one symmetry $s \in A$ (i.e., sps $=q$ ).

(iii) If $\mathcal{A}$ is a purely infinite, simple $C^{*}$-algebra and $p, q \in \mathcal{P}(\mathcal{A})$ are equivalent, then there exist two symmetries $s, s^{\prime}$ in $\tilde{\mathcal{A}}$ such that $s^{\prime}$ spss $s^{\prime}=q$. If two equivalent projections $p, q \in \mathcal{P}(\mathcal{A})$ satisfy $p<q$ or $q<p$, then $p$ and $q$ can not be conjugated by one symmetry.

Proof. (i) Write the $2 \times 2$ matrix form of $p$ with respect to the decomposition $q \oplus(1-q)=1$ as in the proof of Lemma 4. By [Zh3, 2.1] for each positive number $\delta<1-\frac{1}{\sqrt{2}}$ there exists a projection $p_{0}$ such that

$$
\left\|p-p_{0}\right\|<\delta
$$

where $p_{0}$ has a matrix form as explained in the proof of Lemma 4(i). In the following we adopt all notations in the proof of Lemma 4(i). If $\delta$ is small enough, it is well known that there exists a unitary $u_{\epsilon} \in U(\mathcal{A})$ such that

$$
\left\|u_{\epsilon}-1\right\|<\epsilon \quad \text { and } \quad u_{\epsilon} p u_{\epsilon}^{*}=p_{0} \quad([\mathbf{E f}]) .
$$

On the other hand, there exists, by Lemma 2, a symmetry $s^{\prime}$ such that $s^{\prime} p s^{\prime}=p_{0}$.

Since $\sigma(a) \cup \sigma(c) \subset[0,1)$, one sees that

$$
\begin{aligned}
& \|a\|<1, \quad\|f-a\|<1 \\
& \|c\|<1, \quad\|e-c\|<1
\end{aligned}
$$

Applying Lemma 1, one has

$$
\left\|\left(\begin{array}{cc}
a & b \\
b^{*} & c
\end{array}\right)-f\right\|<1, \quad\left\|\left(\begin{array}{cc}
a & b \\
b^{*} & c
\end{array}\right)-e\right\|<1 .
$$

By Lemma 2 there exists a symmetry $s_{0}$ of $(e \oplus f) \mathcal{A}(e \oplus f)$ such that

$$
s_{0} f s_{0}=\left(\begin{array}{cc}
a & b \\
b^{*} & c
\end{array}\right) .
$$

By the equivalence $q \sim p \sim p_{0}$ and the cancellation property of $\mathcal{A}$ one sees

$$
q-f_{0}-f \sim e_{0} .
$$

Let $v_{0}$ be a partial isometry in $\mathcal{A}$ such that

$$
v_{0} v_{0}^{*}=e_{0} \quad \text { and } \quad v_{0}^{*} v_{0}=q-f_{0}-f .
$$


Since $e_{0}\left(q-f_{0}-f\right)=0$, then $s_{1}:=v_{0}+v_{0}^{*}$ is a symmetry in $\left(e_{0} \oplus\left(q-f_{0}-\right.\right.$ $f)) \mathcal{A}\left(e_{0} \oplus\left(q-f_{0}-f\right)\right)$ such that

$$
s_{1}\left(q-f_{0}-f\right) s_{1}=e_{0} .
$$

Set $s:=s_{0} \oplus s_{1} \oplus f_{0} \oplus\left(1-q-e-e_{0}\right)$. Then $s$ is a symmetry of $\tilde{\mathcal{A}}$ such that $s p_{0} s=q$. Then one can choose either a unitary $u_{\epsilon}$ which is close to the identity within $\epsilon$ in norm or a symmetry $s^{\prime}$ such that $u_{\epsilon} p u_{\epsilon}^{*}=p_{0}$ and $s^{\prime} p s^{\prime}=p_{0}$, respectively. It follows that either $s u_{\epsilon} p u_{\epsilon}^{*} s=q$ or $s s^{\prime} p s^{\prime} s=q$, as desired.

(ii) One needs only to consider each summand which is either a full $n \times n$ matrix algebra over complex numbers or $\mathcal{K}$. For either case $q p q$ and $(1-$ $q) p(1-q)$ have discrete spectra. Then $p$ can be replaced by $p_{0}$, as the proof of (i) above indicates. Using the same proof of (i), one obtains a symmetry $s$ of $\tilde{\mathcal{A}}$ such that $s p s=q$. We leave the details to the reader.

(iii) Assume that $\mathcal{A}$ is a purely infinite simple $\mathrm{C}^{*}$-algebra. Separate the proof according to the two cases: $p q=q p$ and $p q \neq q p$.

Case 1. $p q=q p$.

The $2 \times 2$ matrix form of $p$ with respect to the decomposition $(1-q) \oplus q=1$ has two possibilities as follows:

$$
p=\left(\begin{array}{cc}
1-q & 0 \\
0 & p^{\prime}
\end{array}\right), \quad \text { or } \quad p=\left(\begin{array}{cc}
p^{\prime \prime} & 0 \\
0 & p^{\prime}
\end{array}\right), \quad \text { where } p^{\prime \prime}<1-q .
$$

The first possibility above (i.e., $1-q \leq p$ ) can happen only if $\mathcal{A}$ is unital.

First, consider the possibility: $1-q \leq p$. In this case there exists one symmetry $s \in \tilde{\mathcal{A}}$ such that $s p s=q$. In fact, since $p \sim q$ and $p, q \neq 0,1$, the two projections $p$ and $q$ are unitarily equivalent ([EK, 2.4] or [Zh7, Part II]). Then there exists a unitary $u$ of $\tilde{\mathcal{A}}$ such that $u q u^{*}=p$. Write the matrix form of $u$ with respect to $(1-q) \oplus q=1$ as follows:

$$
u:=\left(\begin{array}{cc}
z & x_{1} \\
x_{2} & y
\end{array}\right) \text {. }
$$

By a simple calculation one sees that

$$
x_{1} x_{1}^{*}=1-q, \quad z z^{*}+x_{1} x_{1}^{*}=1-q .
$$

It follows that $z=0$. Set

$$
s=\left(\begin{array}{cc}
0 & x_{2}^{*} \\
x_{2} & y y^{*}
\end{array}\right)
$$


Then $s$ is a symmetry of $\tilde{\mathcal{A}}$ satisfying

$$
s u=\left(\begin{array}{cc}
1-q & 0 \\
0 & x_{2} x_{1}+y
\end{array}\right) .
$$

(The reader is referred to $[\mathbf{Z h} 10,2.1]$ where the construction of $s$ was originated.) Then,

$$
q=s u q u^{*} s=s p s .
$$

Secondly, consider the other possibility: $p=\left(\begin{array}{cc}p^{\prime \prime} & 0 \\ 0 & p^{\prime}\end{array}\right)$. By a standard argument for infinite simple $\mathrm{C}^{*}$-algebras $[\mathbf{C u} 2,1.5]$, there is a projection $q^{\prime}<1-q-p^{\prime \prime}$ such that $p \sim q^{\prime}$ and $q \sim q^{\prime}$. Let $v_{1} \in \mathcal{A}$ be a partial isometry such that

$$
v_{1} v_{1}^{*}=p \quad \text { and } \quad v_{1}^{*} v_{1}=q^{\prime} .
$$

Set $s:=v_{1}+v_{1}^{*}+\left(1-p-q^{\prime}\right)$. Then $s$ is a symmetry such that $s p s=q^{\prime}$. Similarly, there exists another symmetry $s^{\prime}$ in $\tilde{\mathcal{A}}$ such that $s^{\prime} q^{\prime} s^{\prime}=q$. Hence,

$$
s^{\prime} s p s s^{\prime}=q .
$$

If $q<p$ (or $p<q$ ) and $p \sim q$, then one symmetry is not enough to conjugate $p$ and $q$ (We are indebted to N.C. Phillips for pointing out to us that this observation also follows from $[\mathrm{Br}]$.) In fact, if there were one symmetry, say $2 r-1$ or $1-2 r$ for some projection $r \in \mathcal{A}$, such that

$$
(2 r-1) q(2 r-1)=p
$$

then $q-p=2 r q(1-r)+2(1-r) q r$. With respect to the decomposition $r \oplus(1-r)=1$, the nonzero projection $q-p$ has the form

$$
q-p=\left(\begin{array}{cc}
0 & 2(1-r) q r \\
2 r q(1-r) & 0
\end{array}\right)
$$

which is impossible.

Case 2. $q p \neq p q$.

Clearly, $q p(1-q) \neq 0$, and hence $0<\delta:=\|q p q-q\| \leq 1$. Furthermore, $q p q$ is not a projection, since $q p q-(q p q)^{2}=q p(1-q) p q \neq 0$. Using the proof of (i), one gets a projection $p_{0}$ such that

$$
\left\|p-p_{0}\right\|<\frac{\delta_{0}}{4}
$$


where $p_{0}$ has a matrix form as explained in the proof of Lemma 4(i). It follows that

$$
\left\|q p q-\left(\begin{array}{cc}
f_{0} & 0 \\
0 & a
\end{array}\right)\right\|<\frac{\delta_{0}}{4}
$$

Then

$$
\left\|\left(\begin{array}{cc}
f_{0} & 0 \\
0 & a
\end{array}\right)-q\right\| \geq\|q-q p q\|-\left\|q p q-\left(\begin{array}{cc}
f_{0} & 0 \\
0 & a
\end{array}\right)\right\|>\frac{3 \delta_{0}}{16} .
$$

There are two possibilities: either $f_{0} \oplus f<q$ or $f_{0} \oplus f=q$.

Consider the first possibility: $f_{0} \oplus f<q$. Then, by Lemma 4(i), there exists a symmetry $s \in \tilde{\mathcal{A}}$ such that

$$
\text { sps }=p_{1} \oplus p_{2}, \quad \text { where } \quad p_{1} \leq f_{0} \oplus f \quad \text { and } \quad p_{2} \leq 1-q .
$$

Then sps and $q$ are equivalent projections in the unital hereditary $\mathrm{C}^{*}$ subalgebra

$$
\left(q \oplus p_{2}\right) \mathcal{A}\left(q \oplus p_{2}\right) ;
$$

notice that this hereditary $\mathrm{C}^{*}$-subalgebra is again a purely infinite simple $\mathrm{C}^{*}$-algebra. Furthermore, sps commutes with $q$. Applying the proof of Case 1 above, one obtains a symmetry $s_{0}$ of $\left(q \oplus p_{2}\right) \mathcal{A}\left(q \oplus p_{2}\right)$ such that

$$
s_{0} s p s s_{0}=q .
$$

Set $s^{\prime}:=s_{0} \oplus\left(1-q-p_{2}\right)$. Then $s^{\prime}$ is a symmetry in $\tilde{\mathcal{A}}$ such that

$$
s^{\prime} s p s s^{\prime}=q
$$

Now consider the other possibility: $f_{0} \oplus f=q$. From the construction of $a$ (in the proof of Lemma 4(i)) and the estimate above, one has

$$
\left\|q-\left(\begin{array}{cc}
f_{0} & 0 \\
0 & a
\end{array}\right)\right\|=\left\|\sum_{i=1}^{m}\left(1-t_{i}\right) v r_{i} v^{*}\right\|>\frac{3 \delta_{0}}{4} .
$$

Then there is at least one $i$ such that $1-t_{i}>\frac{3 \delta_{0}}{4} ; t_{i}<1-\frac{3 \delta_{0}}{4}$ for such an $i$. Set

$$
r_{0}^{\prime}=\left(\begin{array}{ccccc}
f_{0} & 0 & 0 & 0 \\
0 & \sum_{\left\{i: t_{i}>1-\frac{3 \delta_{0}}{4}\right\}} v r_{i} v^{*} & 0 & 0 \\
0 & 0 & \sum_{\left\{i: t_{i} \leq 1-\frac{3 \delta_{0}}{4}\right\}} r_{i} & 0 \\
& & 0 & e_{0}
\end{array}\right) .
$$


From a simple calculation and the construction of $v$ (in the proof of Lemma 4(i)) one sees that

$$
\begin{aligned}
\left\|r_{0}^{\prime}-r_{0}^{\prime} p_{0} r_{0}^{\prime}\right\| & \leq\left\|\left(\begin{array}{cccc}
0 & 0 & 0 & 0 \\
0 & \sum_{\left\{i: t_{i}>1-\frac{3 \delta_{0}}{4}\right\}}\left(1-t_{i}\right) v r_{i} v^{*} & 0 & 0 \\
0 & 0 & \sum_{\left\{i: t_{i} \leq 1-\frac{3 \delta_{0}}{4}\right\}} t_{i} r_{i} & 0 \\
0 & 0 & 0
\end{array}\right)\right\| \\
& \leq \max \left\{\frac{3 \delta_{0}}{4}, 1-\frac{3 \delta_{0}}{4}\right\}:=\delta_{1} .
\end{aligned}
$$

Similarly, one has

$$
\left\|\left(1-r_{0}^{\prime}\right) p_{0}\left(1-r_{0}^{\prime}\right)\right\| \leq \delta_{1} .
$$

Then Lemma 1 and the estimate above imply

$$
\left\|r_{0}^{\prime}-p_{0}\right\| \leq \sqrt{\delta_{1}}
$$

Thus,

$$
\left\|p-r_{0}^{\prime}\right\| \leq\left\|p-p_{0}\right\|+\left\|p_{0}-r_{0}^{\prime}\right\| \leq \frac{\delta_{0}}{16}+\sqrt{\delta_{1}}<1 .
$$

By Lemma 2 there is a symmetry $s$ in $\tilde{\mathcal{A}}$ such that $s p s=r_{0}^{\prime}$. Noticing that

$$
f_{0} \oplus \sum_{\left\{i: t_{i}>1-\frac{3 \delta_{0}}{4}\right\}} v r_{i} v^{*}<q,
$$

we can once again apply the proof of the case 1 above to get another symmetry $s^{\prime}$ of $\tilde{\mathcal{A}}$ such that

$$
s^{\prime} \operatorname{sps}^{\prime}=q .
$$

Hence, the conclusion (iii) holds. The proof is complete.

Corollary 6. Suppose that $\mathcal{A}$ is a $C^{*}$-algebra with $R R(\mathcal{A})=0$.

(i) If $\mathcal{A}$ has cancellation, then $\sup _{p, q}\left\{d_{r}(p, q): p \approx q\right.$ in $\left.\mathcal{P}(\mathcal{A})\right\} \leq \frac{\pi}{2}$.

(ii) If $\mathcal{A}$ is purely infinite and simple, then $\sup _{p, q}\left\{d_{r}(p, q): p \approx q\right.$ in $\mathcal{P}(\mathcal{A})\} \leq \pi$

Proof. (i) Observe first the following fact: If a rectifiable path $\{u(t): 0 \leq$ $t \leq 1\}$ of unitaries in $\tilde{\mathcal{A}}$ has length $l$, then the length $l_{0}$ of the corresponding rectifiable path $\left\{u(t) p u(t)^{*}: 0 \leq t \leq 1\right\}$ in $\mathcal{P}(\mathcal{A})$ is less than or equal to $l$ (this statement is valid for any $\mathrm{C}^{*}$-algebra). A proof of this statement is given in $[\mathbf{P h} 1]$, but the following one seems to be much simpler. It suffices to show that

$$
\sum_{i=1}^{n}\left\|u\left(t_{i}\right) p u\left(t_{i}\right)^{*}-u\left(t_{i-1}\right) p u\left(t_{i-1}\right)^{*}\right\| \leq \sum_{i=1}^{n}\left\|u\left(t_{i}\right)-u\left(t_{i-1}\right)\right\|
$$


for any subdivision of [0,1]: $0=t_{0}<t_{1}<t_{2}<\ldots<t_{n}=1$. But the last inequality follows from the following estimate:

$$
\begin{aligned}
& \left\|u\left(t_{i}\right) p u\left(t_{i}\right)^{*}-u\left(t_{i-1}\right) p u\left(t_{i-1}\right)^{*}\right\| \\
& =\left\|p\left(u\left(t_{i}\right)^{*} u\left(t_{i-1}\right)-1\right)-\left(u\left(t_{i}\right)^{*} u\left(t_{i-1}\right)-1\right) p\right\| \\
& \leq \max \left\{\left\|p\left(u\left(t_{i}\right)^{*} u\left(t_{i-1}\right)-1\right)(1-p)\right\|,\left\|(1-p)\left(u\left(t_{i}\right)^{*} u\left(t_{i-1}\right)-1\right) p\right\|\right\} \\
& \leq\left\|u\left(t_{i}\right)^{*} u\left(t_{i-1}\right)-1\right\| \\
& =\left\|u\left(t_{i}\right)-u\left(t_{i-1}\right)\right\| .
\end{aligned}
$$

Assume that $\mathcal{A}$ has cancellation and $p, q$ are any two homotopic projections in $\mathcal{P}(\mathcal{A})$. By Theorem 5(i) there exist for any number $\epsilon>0$ a symmetry $s$ and a unitary $u_{\epsilon}$ in $\tilde{\mathcal{A}}$ such that $s u_{\epsilon} p u_{\epsilon}^{*} s=q$. Then $(-i s) u_{\epsilon} p u_{\epsilon}^{*}(i s)=q$. Let $r$ be the spectral projection of $s$ associated with $1 \in \sigma(s)$. Then the rectifiable path

$$
\left\{u(\theta):=e^{\theta i} r+e^{-\theta i}(1-r): 0 \leq \theta \leq \frac{\pi}{2}\right\}
$$

has a length $\frac{\pi}{2}$. This path joins $i s$ and the identity in the unitary group of $\tilde{\mathcal{A}}$. Using the observation above, one sees that the rectifiable path

$$
\left\{u(\theta) p u(\theta)^{*}: 0 \leq \theta \leq \frac{\pi}{2}\right\} \subset \mathcal{P}(\mathcal{A})
$$

joins $p$ and $q$ and has a length $\frac{\pi}{2}$. Then $d_{r}(p, q) \leq \frac{\pi}{2}$. Since $p$ and $q$ are any two homotopic projections in $\mathcal{P}(\mathcal{A})$, the conclusion (i) follows, i.e.,

$$
\sup _{p, q}\left\{d_{r}(p, q): p \approx q \text { in } \mathcal{P}(\mathcal{A})\right\} \leq \frac{\pi}{2} .
$$

(ii) If $s_{1}, s_{2}, \ldots, s_{m}$ are symmetries in $\tilde{\mathcal{A}}$ such that

$$
s_{1} s_{2} \ldots s_{m} p s_{m} \ldots s_{2} s_{1}=q,
$$

then $\left(-i s_{1}\right)\left(-i s_{2}\right) \ldots\left(-i s_{m}\right) p\left(i s_{m}\right) \ldots\left(i s_{2}\right)\left(i s_{1}\right)=q$. The rectifiable path obtained by joining recursively all $-i s_{k}(1 \leq k \leq m)$ to the identity has a length $\frac{m \pi}{2}([\mathbf{P h} \mathbf{1}])$. Take $m=2$. Then the conclusion (ii) follows from Theorem 5 (iii).

Proposition 7. Two equivalent projections $p$ and $q$ in any stable $C^{*}$-algebra $\mathcal{A} \otimes \mathcal{K}$ are conjugated by a product of three symmetries, and also by a product of two symmetries and a unitary which is arbitrarily close in norm to the identity (of the unitization $\left.(\mathcal{A} \otimes \mathcal{K})^{\dagger}\right)$. As a consequence,

$$
\sup _{p, q}\left\{d_{r}(p, q): p \approx q \text { in } \mathcal{P}(\mathcal{A} \otimes \mathcal{K})\right\} \leq \pi
$$


Proof. Given any number $\epsilon>0$. There are two projections $p^{\prime}$ and $q^{\prime}$ in $M_{n}(\mathcal{A})$ for some $n$ with

$$
\left\|p^{\prime}-p\right\|<\delta \quad \text { and } \quad\left\|q^{\prime}-q\right\|<\delta
$$

Let $\delta$ be small enough so that one can choose unitaries $u_{1}$ and $u_{2}$ in $(\mathcal{A} \otimes \mathcal{K})^{\dagger}$ which are close to the identity within $\epsilon / 2$ and satisfy

$$
u_{1} p u_{1}^{*}=p^{\prime} \quad \text { and } \quad u_{2} q u_{2}^{*}=q^{\prime} .
$$

One can also choose two symmetries $s_{1}$ and $s_{2}$ such that

$$
s_{1} p^{\prime} s_{1}=s_{2} q^{\prime} s_{2} \leq 1-\sum_{i=1}^{n} 1 \otimes e_{i i}
$$

where $\sum_{i=1}^{n} 1 \otimes e_{i i}$ is the identity of $M_{n}(\tilde{\mathcal{A}})$. It follows that

$$
u_{2}^{*} s_{2} s_{1} u_{1} p u_{1}^{*} s_{1} s_{2} u_{2}=q .
$$

Rewrite $u_{2}^{*} s_{2} s_{1} u_{1}=\left(u_{2}^{*} s_{2} u_{2}\right)\left(u_{2}^{*} s_{1} u_{2}\right)\left(u_{2}^{*} u_{1}\right)$ and $\left\|u_{2}^{*} u_{1}-1\right\|<\epsilon$, and set

$$
s_{1}^{\prime}=u_{2}^{*} s_{1} u_{2}, \quad s_{2}^{\prime}=u_{2}^{*} s_{2} u_{2}, \quad \text { and } \quad u_{\epsilon}=u_{2}^{*} u_{1} .
$$

Notice $\left\|u_{2}^{*} u_{1} p u_{1}^{*} u_{2}-p\right\|<2 \epsilon<1$ (if $\epsilon<\frac{1}{2}$ ). By Lemma 2 one can choose another symmetry $s_{3}$ such that

$$
s_{2}^{\prime} s_{1}^{\prime} s_{3} p s_{3} s_{1}^{\prime} s_{2}^{\prime}=q .
$$

The consequence follows from the same proof as of Corollary 6(ii).

L.G. Brown has investigated whether a minimizing path joining two homotopic projections exists in $\mathcal{L}(\mathcal{H})$ (and he also pointed out that his techniques work for all von Neumann algebras $[\mathrm{Br}, 2.12,3.3])$. One of his results asserts that the range of $d_{r}(p, q)$ is the whole interval $[0, \pi]$, and consequently, $\sup _{p, q}\left\{d_{r}(p, q): p \approx q\right.$ in $\left.\mathcal{P}(\mathcal{A})\right\}=\pi$. The following theorem gives an estimate for the number of symmetries conjugating two homotopic projections in $\mathcal{L}(\mathcal{H})$ and in a type $\mathrm{II}_{\infty}$ factor, which in turn yields an estimate for the projective length (covered by the result of L.G. Brown above). Here we point out that our work only employs $\mathrm{C}^{*}$-algebra techniques.

Theorem 8. Suppose that $\mathcal{M}$ is either $\mathcal{L}(\mathcal{H})$ or a type $\mathrm{II}_{\infty}$ factor and $\epsilon$ is an arbitrary positive number. Then two projections $p$ and $q$ of $\mathcal{M}$ are homotopic if and only if there exist two symmetries $s_{1}, s_{2}$ and a unitary $u_{\epsilon}$ such that

$$
\left\|u_{\epsilon}-1\right\|<\epsilon \quad \text { and } \quad s_{1} s_{2} u_{\epsilon}^{*} p u_{\epsilon} s_{2} s_{1}=q
$$


again if and only if there exist at most three symmetries $s_{1}, s_{2}$ and $s_{3}$ such that

$$
s_{1} s_{2} s_{3} p s_{3} s_{2} s_{1}=q
$$

Proof. Let $p$ and $q$ be two homotopic projections in either a type $\mathrm{II}_{\infty}$ factor or in $\mathcal{L}(\mathcal{H})$. Then a projection $r$ in these algebras is finite if and only if $r$ has a finite trace value. If both $p$ and $q$ are finite projections, then $p$ and $q$ are homotopic as long as they are equivalent. Applying the same arguments as in the proof of Theorem 5 (i) to $p$ and $q$, one gets two symmetries $s_{1}$ and $s_{2}$ such that $s_{2} s_{1} p s_{1} s_{2}=q$. If both $1-p$ and $1-q$ are finite homotopic projections, then there exist two symmetries $s_{1}$ and $s_{2}$ of $\mathcal{M}$ such that

$$
s_{2} s_{1}(1-p) s_{1} s_{2}=1-q, \quad \text { and hence } \quad s_{2} s_{1} p s_{1} s_{2}=q .
$$

Assume that all $p, q, 1-p$, and $1-q$ are infinite projections. As in the proof of Theorem 5(iii) we separate the discussion into two cases: either $p q=q p$ or $p q \neq q p$.

Case 1. $p q=q p$.

Two possibilities of the matrix form of $p$ with respect to the decomposition $(1-q) \oplus q=1$ are as follows:

$$
p=\left(\begin{array}{cc}
1-q & 0 \\
0 & p^{\prime}
\end{array}\right) \quad \text { or } \quad p=\left(\begin{array}{cc}
p^{\prime \prime} & 0 \\
0 & p^{\prime}
\end{array}\right), \quad \text { where } p^{\prime \prime}<1-q .
$$

For the first possibility $1-q \leq p$ there exists one symmetry $s$ such that $s p s=q$ by the same arguments as in the proof of Theorem 5 (iii). For the second possibility $p^{\prime \prime}<1-q$ there are two situations: $1-q-p^{\prime \prime}$ is either finite or infinite. If $1-q-p^{\prime \prime}$ is infinite, then the same arguments in the proof of Theorem 5 (iii) apply. If $1-q-p^{\prime \prime}$ is finite, then $q-p^{\prime}$ must be infinite. By considering the hereditary subalgebra supported by $\left(q \oplus p^{\prime \prime}\right)$ instead, one reduces the problem to the first possibility.

Case 2. $\quad q p \neq p q$.

As in the proof of Lemma 4(i) we approximate $p$ within $\delta$ in norm by a projection

$$
p_{0}=\left(\begin{array}{cccc}
f_{0} & 0 & 0 & 0 \\
0 & a & b & 0 \\
0 & b^{*} & c & 0 \\
0 & 0 & 0 & e_{0}
\end{array}\right) .
$$

If $\delta$ is small enough, then there exist a unitary $u_{\epsilon}$ and a symmetry $s_{3}$ such that $u_{\epsilon} p u_{\epsilon}^{*}=p_{0}$ and $s_{3} p s_{3}=p_{0}$. It suffices to show that the product of two symmetries conjugates $p_{0}$ and $q$. 
If $e \oplus e_{0}=1-q$ where $e$ is the projection onto the range of $c$, then there exists one symmetry $s_{2}$ conjugating $p_{0}$ and

$$
p_{1}=\left(\begin{array}{cccc}
f_{0} & 0 & 0 & 0 \\
0 & 0 & 0 & 0 \\
0 & 0 & e & 0 \\
0 & 0 & 0 & e_{0}
\end{array}\right),
$$

where the construction of $s_{2}$ is given in the proof of Lemma 4(i). Then $1-q \leq$ $p_{1}$, and hence, the arguments of Case 1 above apply. Thus, there exists another symmetry $s_{1}$ such that $s_{1} p_{1} s_{1}=q$. Therefore, either $s_{1} s_{2} u_{\epsilon}^{*} p u_{\epsilon} s_{2} s_{1}=$ $q$ or $s_{1} s_{2} s_{3} p s_{3} s_{2} s_{1}=q$.

If $e \oplus e_{0}<1-q$, then there are two possibilities: $1-q-e-e_{0}$ is either finite or infinite. If $1-q-e-e_{0}$ is finite, then one works with the hereditary subalgebra supported by $q \oplus e \oplus e_{0}$ instead. In this way the problem is reduced to what we just discussed in the last paragraph. If $1-q-e-e_{0}$ is infinite, then one can choose a symmetry $s_{2}$ such that $s_{2} p_{0} s_{2}<1-q-e-e_{0}$, and then, choose another symmetry $s_{1}$ such that $s_{1} s_{2} p_{0} s_{2} s_{1}=q$. The proof is complete.

Recall that $\mathcal{L}\left(\mathcal{H}_{\mathcal{A}}\right)$ is the $\mathrm{C}^{*}$-algebra of all bounded operators (with adjoints) on a Hilbert $\mathrm{C}^{*}$-module $\mathcal{H}_{\mathcal{A}}$ associated with a $\mathrm{C}^{*}$-algebra $\mathcal{A}([\mathbf{K s}])$. It is well known that $\mathcal{L}\left(\mathcal{H}_{\mathcal{A}}\right) \cong M(\mathcal{A} \otimes \mathcal{K})$, where $M(\mathcal{A} \otimes \mathcal{K})$ is the multiplier algebra of $\mathcal{A} \otimes \mathcal{K}([\mathrm{Bl}, 13.4 .1])$. Consider the following two classes of $\mathrm{C}^{*}$-algebras:

(1) All $\mathrm{C}^{*}$-algebras with the following property: There is a map $\tau$ from the semi-group $D(\mathcal{A} \otimes \mathcal{K})$ consisting of equivalence classes of projections in $\mathcal{A} \otimes \mathcal{K}$ to the non-negative numbers such that

$$
\begin{aligned}
& \text { (i) } \tau\left(\left[r_{1}\right]+\left[r_{2}\right]\right)=\tau\left(\left[r_{1}\right]\right)+\tau\left(\left[r_{2}\right]\right), \\
& \text { (ii) }[p] \leq[q] \quad \text { whenever } \tau([p])<\tau([q]) .
\end{aligned}
$$

(2) All $\sigma$-unital, purely infinite, simple $\mathrm{C}^{*}$-algebras.

Theorem 9. Suppose that $R R\left(\mathcal{L}\left(\mathcal{H}_{\mathcal{A}}\right)\right)=0$ and $\mathcal{A}$ is a $\sigma$-unital $C^{*}$-algebra in either class (1) or in class (2) above. Then two projections $p$ and $q$ of $\mathcal{L}\left(\mathcal{H}_{\mathcal{A}}\right)$ are homotopic if and only if there exist two symmetries $s_{1}, s_{2}$ and a unitary $u_{\epsilon}$ such that

$$
\left\|u_{\epsilon}-1\right\|<\epsilon \quad \text { and } \quad s_{1} s_{2} u_{\epsilon}^{*} p u_{\epsilon} s_{2} s_{1}=q ;
$$

again if and only if there exist at most three symmetries $s_{1}, s_{2}$ and $s_{3}$ such that

$$
s_{1} s_{2} s_{3} p s_{3} s_{2} s_{1}=q \text {. }
$$


Proof. If $\mathcal{A}$ is a $\mathrm{C}^{*}$-algebra in the class (1), one can naturally extend $\tau$ on $D\left(\mathcal{L}\left(\mathcal{H}_{\mathcal{A}}\right)\right)$ with values in $[0,+\infty]$. In fact, for each projection $p \in \mathcal{L}\left(\mathcal{H}_{\mathcal{A}}\right)$ choose an approximate identity of $p(\mathcal{A} \otimes \mathcal{K}) p$ consisting of a sequence of projections, say $\left\{e_{n}\right\}$, such that $e_{n}<e_{n+1}$ for any $n \geq 1$ ([Zh6, 1.2]). Define

$$
\tau([p])=\lim \tau\left(\left[e_{n}\right]\right) .
$$

All projections in $\mathcal{L}\left(\mathcal{H}_{\mathcal{A}}\right)$ such that $\tau([p])<\infty$ generate a closed ideal $J$ of $\mathcal{L}\left(\mathcal{H}_{\mathcal{A}}\right)$. Then one can prove by the Riesz decomposition property [Zh2] that $p \in J$ if and only if $\tau([p])<\infty$; and $\tau([p])=\infty$ if and only if $p \sim 1$ $([\operatorname{Lin} 1])$. Under the assumption that $R R\left(\mathcal{L}\left(\mathcal{H}_{\mathcal{A}}\right)\right)=0$, the conclusion follows from exactly the same arguments as in the proof of Theorem 8 for type $\mathrm{II}_{\infty}$ factors. The details are left to the reader.

Assume that $\mathcal{A}$ is a $\mathrm{C}^{*}$-algebra in the class (2). Then $R R\left(\mathcal{L}\left(\mathcal{H}_{\mathcal{A}}\right)\right)=0$ if and only if $K_{1}(\mathcal{A})=0([\mathbf{Z h} 7$, Part I $])$. Assume that $p$ and $q$ are two homotopic projections in $\mathcal{L}\left(\mathcal{H}_{\mathcal{A}}\right)$. Since $R R\left(\mathcal{L}\left(\mathcal{H}_{\mathcal{A}}\right)\right)=0$, the construction in the proof of $[\mathbf{Z h 3}, 2.1]$ applies to $p$ and $q$. Using the fact $[\mathbf{Z h 2}, 3.3]$ that every projection of $\mathcal{L}\left(\mathcal{H}_{\mathcal{A}}\right)$ not in $\mathcal{A} \otimes \mathcal{K}$ is equivalent to the identity, we can apply the same arguments as in the proof of Theorem 8 for $\mathcal{L}(\mathcal{H})$, where the condition whether a projection is finite or infinite is replaced by whether a projection is in $\mathcal{A} \otimes \mathcal{K}$ or in $\mathcal{L}\left(\mathcal{H}_{\mathcal{A}}\right) \backslash \mathcal{A} \otimes \mathcal{K}$. We leave it to the reader to fill the details. Here the reader may need the well known fact that $1-r \sim 1$ in $\mathcal{L}\left(\mathcal{H}_{\mathcal{A}}\right)$ whenever $r$ is a projection in $\mathcal{A} \otimes \mathcal{K}([\mathbf{C u} 3,3.6])$.

Remark. Specific examples satisfying the hypotheses of Theorem 9 are matroid algebras [El1], type $\mathrm{II}_{1}$ factors, simple $\mathrm{AF}$ algebras with unique trace up to multiples ([Lin2]), the Cuntz algebras $\mathcal{O}_{n} 2 \leq 2 \leq+\infty$, and type III factors [Zh7, Part I].

Corollary 10. Let $\mathcal{A}$ be a $C^{*}$-algebra satisfying the hypotheses of Theorem 9. Then

$$
\sup _{p, q}\left\{d_{r}(p, q): p \approx q \in \mathcal{P}(M(\mathcal{A} \otimes \mathcal{K}))\right\} \leq \pi
$$

Proof. The estimate follows immediately from Theorem 9 and the proof of Corollary 6.

\section{Theorem 11.}

(i) Let $\mathcal{B}$ be a unital $C^{*}$-algebra and $\mathcal{A}$ be a $\sigma$-unital $C^{*}$-algebra. Then every unitary of $\mathcal{B} \otimes \mathcal{L}\left(\mathcal{H}_{\mathcal{A}}\right)$ can be written as a product of at most seven symmetries.

(ii) If $\mathcal{D}$ is a purely infinite, simple $C^{*}$-algebra and $X$ is any compact Hausdorff space, then every unitary in the identity path component of 
the unitary group of $C(X, \tilde{\mathcal{D}})$ can be approximated by some products of six symmetries.

(iii) Two homotopic projections in $\mathcal{B} \otimes \mathcal{L}\left(\mathcal{H}_{\mathcal{A}}\right)$ can be conjugated by a product of at most six symmetries and a unitary which is arbitrarily close to the identity in norm, and can also be conjugated by a product of at most seven symmetries. The same conclusions hold also for any two homotopic projections in $C(X, \tilde{\mathcal{D}})$.

(iv) If $\mathcal{E}$ is a $C^{*}$-algebra of the form $\mathcal{B} \otimes \mathcal{L}\left(\mathcal{H}_{\mathcal{A}}\right)$ or the form $C(X, \tilde{\mathcal{D}})$, then

$$
\sup _{p, q}\left\{d_{r}(p, q): p \approx q \in \mathcal{P}(\mathcal{E})\right\} \leq 3 \pi .
$$

Proof. A proof of (i) and (ii) can be found as a part of the proof of [Zh10] (there for other purposes). The conclusion (iii) is an immediate corollary of (i) and (ii); and the estimate (iv) is a corollary of (iii) and the proof of Corollary 6.

Corollary 12. Let $\mathcal{A}, \mathcal{B}, \mathcal{D}$, and $X$ be the same as in Theorem 11 . Then $\mathcal{B} \otimes \mathcal{L}\left(\mathcal{H}_{\mathcal{A}}\right)$ is generated algebraically by projections, and $C(X, \tilde{\mathcal{D}})$ is generated by projections.

Proof. It is well known that every element of a unital $\mathrm{C}^{*}$-algebra is a linear combination of four unitaries [KR, I(4.1.7)]. Furthermore, these unitaries can be chosen from the identity path component of the unitary group by the proof of $[\mathbf{K R}, \mathrm{I}]$. Theorem 11 asserts that every unitary of $\mathcal{B} \otimes \mathcal{L}\left(\mathcal{H}_{\mathcal{A}}\right)$ can be written as a product of seven symmetries

$$
\left(2 r_{1}-1\right)\left(2 r_{2}-1\right) \ldots\left(2 r_{7}-1\right)
$$

where $r_{1}, r_{2}, \ldots, r_{7}$ are projections of $\mathcal{B} \otimes \mathcal{L}\left(\mathcal{H}_{\mathcal{A}}\right)$. Hence, $\mathcal{B} \otimes \mathcal{L}\left(\mathcal{H}_{\mathcal{A}}\right)$ is generated algebraically by projections. Similarly, since every unitary in the identity path component of the unitary group of $C(X, \tilde{\mathcal{D}})$ can be approximated by products of six symmetries, the algebra $C(X, \tilde{\mathcal{D}})$ is generated by projections.

Corollary 13. Suppose that $\mathcal{A}$ is any $\sigma$-unital $C^{*}$-algebra and $X$ is any compact Hausdorff space. Then the following hold:

(i) Both $M(\mathcal{A} \otimes \mathcal{K})$ and $C\left(X, \mathcal{L}\left(\mathcal{H}_{\mathcal{A}}\right)\right)$ are generated by projections.

(ii) If $\mathcal{E}$ is either $M(\mathcal{A} \otimes \mathcal{K})$ or $C\left(X, \mathcal{L}\left(\mathcal{H}_{\mathcal{A}}\right)\right)$, then

$$
\sup _{p, q}\left\{d_{r}(p, q): p \approx q \in \mathcal{P}(\mathcal{E})\right\} \leq 3 \pi .
$$


Proof. In Corollary 11, if one takes $\mathcal{B}$ to be the algebra of complex numbers, then $\mathcal{B} \otimes \mathcal{L}\left(\mathcal{H}_{\mathcal{A}}\right) \cong M(\mathcal{A} \otimes \mathcal{K})$. If one takes $\mathcal{B}$ to be $C(X)$, then $\mathcal{B} \otimes \mathcal{L}\left(\mathcal{H}_{\mathcal{A}}\right) \cong$ $C\left(X, \mathcal{L}\left(\mathcal{H}_{\mathcal{A}}\right)\right)$.

\section{References}

[Bl] B. Blackadar, K-theory for operator algebras, Springer-Verlag, New York-BerlinHeidelberg-London-Paris-Tokyo, 1987.

[BK] B. Blackadar and A. Kumjian, Skew products of relations and structure of simple $C^{*}$-algebras, Math. Z., 189 (1985), 55-63.

[BKR] B. Blackadar, A. Kumjian and M. Rørdam, Approximately central matrix units and the structure of non-commutative tori, K-Theory, 6 (1992), 267-284.

[Bre1] M. Breuer, A generalization of Kuiper's theorem to factors of type $\mathrm{II}_{\infty}$, J. Math. Mech., 16 (1967), 917-925.

[Bre2] - On the homotopy type of the group of regular elements of semifinite von Neumann algebras, Math. Ann., 185 (1970), 61-74.

[Br] L.G. Brown, The rectifiable metric on the set of closed subspaces of Hilbert space, Trans. Amer. Math. Soc., to appear.

[BP] L.G. Brown and G.K. Pedersen, $C^{*}$-algebras of real rank zero, J. Funct. Anal., 99 (1991), 131-149.

[BD] J. Bunce and J. Deddens, A family of simple $C^{*}$-algebras related to weighted shift operators, J. Funct. Anal., 19 (1975), 13-24.

[CE] M-D. Choi and G.A. Elliott, Density of the self-adjoint elements with finite spectrum in an irrational rotation $C^{*}$-algebra, Math. Scand., 67 (1990), 73-86.

[Cu1] J. Cuntz, Simple $C^{*}$-algebras generated by isometries, Comm. Math. Phys., 57 (1977), 173-185.

[Cu2] , K-theory for certain $C^{*}$-algebras, Ann. of Math., 131 (1981), 181-197.

[Cu3] - A class of $C^{*}$-algebras and topological Markov Chains II: Reducible Markov chains and the Ext-functor for $C^{*}$-algebras, Invent. Math., 63 (1981), 25-40.

[CK] J. Cuntz and W. Krieger, A class of $C^{*}$-algebras and topological Markov chains, Inventions Math., 56 (1980), 251-268.

[Ef] E.G. Effros, Dimensions and $C^{*}$-algebras, CBMS Regional Conference Series in Mathematics A.M.S, 46 (1981).

[EK] E.G. Effros and J. Kaminker, Some homotopy and shape calculations for $C^{*}$-algebras, Group Representations, Ergodic Theory, Operator Algebras, And Mathematical Physics, MSRI Publication No. 6, Springer Verlag, New York, 1987.

[El1] G.A. Elliott, Derivations of matroid $C^{*}$-algebras II, Ann. of Math., 100 (1974), 407-422.

[El2] The classification of $C^{*}$-algebras of real rank zero, J. Reine Angew. Math., 443 (1993), 179-219.

[EE] G.A. Elliott and D.E. Evans, The structure of irrational rotation $C^{*}$-algebras, Ann. Math., 138 (1993), 477-501.

[G] K.R. Goodearl, Some notes on yet another family of simple $C^{*}$-algebras that sometimes have real rank zero, preprint. 
[HK] P.R. Halmos and S. Kakutani, Products of symmetries, Bull. A.M.S., 64 (1958), 77-78.

[KR] R.V. Kadison and J.R. Ringrose, Fundamentals of the theory of operator algebras, Vol. I, II, Academic Press, Inc., 1986.

[Ks] G.C. Kasparov, Hilbert $C^{*}$-modules: Theorems of Stinespring and Voiculescu, J. Operator Theory, 4 (1980), 133-150.

[Lin1] H. Lin, Ideals of multiplier algebras of simple AF $C^{*}$-algebras, Proc. Amer. Math. Soc., 104 (1988), 239-244.

[Lin2] — Generalized Weyl-von Neumann Theorem, Int. J. Math., 2 (1991), 725-739.

[LZ] H. Lin and S. Zhang, Finite simple $C^{*}$-algebras, J. Funct. Anal., 100 (1991), 221231.

[Pe] G.K. Pedersen, The linear span of projections in simple $C^{*}$-algebras, J. Operator Theory, 4 (1980), 289-296.

[Ph1] N.C. Phillips, The rectifiable metric on the space of projections in a $C^{*}$-algebra, Int. J. Math, 3 (1992), 679-698.

[Ph2] - Approximation by unitaries with finite spectrum in purely infinite simple $C^{*}$-algebras, J. Funct. Anal., 120 (1994), 98-106.

[PR] N.C. Phillips and J.R. Ringrose, Exponential rank in operator algebras, pp. 395 - 413 in: Current Topics in Operator Algebras, H. Araki etc., editors, World Scientific, 1991.

[Ri1] M.A. Rieffel, The cancellation theorem for projective modules over irrational rotation $C^{*}$-algebras, Proc. London Math. Soc., 47 (1983), 285-302.

[Ri2] - The homotopy groups of the unitary groups of non-commutative tori, J. Operator Theory, 17 (1987), 237-254.

[Rin] J.R. Ringrose, Exponential length and exponential rank in $C^{*}$-algebras, preprint.

[Rø] M. Rørdam, Ideals in the multiplier algebra of a stable algebra, J. Operator Theory, to appear.

[Sc] H. Schröder, The homotopy type of the regular group of a Banach algebra (a survey), preprint.

[Ta] M. Takesaki, Theory of Operator Algebras, I, Springer - Verlag, New York-HeidelbergBerlin, 1979.

[Th] K. Thomsen, Non-stable K-theory for operator algebras, I, K-theory, 4 (1991), 227243.

[Zh1] S. Zhang, On the structure of projections and ideals of corona algebras, Can. J. Math., 41 (1989), 721-742.

[Zh2] - A Riesz decomposition property and ideal structure of multiplier algebras, J. Operator Theory, 24 (1990), 209-225.

[Zh3] , Diagonalizing projections in the multiplier algebra and matrices over a $C^{*}$ algebra, Pac. J. Math., 145 (1990), 181-200.

[Zh4] $\quad$ A property of purely infinite simple $C^{*}$-algebras, Proc. Amer. Math. Soc., 109 (1990), 717-720.

[Zh5] Matricial structure and homotopy type of simple $C^{*}$-algebras of real rank zero, J. Operator Theory, 26 (1991), 283-312. 
[Zh6] , K K-groups, quasidiagonality and interpolation by multiplier projections, Trans. Amer. Math. Soc., 325 (1991), 793-818.

[Zh7] , Certain $C^{*}$-algebras with real rank zero and their corona and multiplier algebras, Part I, Pac. J. Math., 155 (1992), 169-197; Part II, K-theory, 6 (1992), $1-27$.

[Zh8] Exponential rank and exponential length of operators on Hilbert $C^{*}$-module, Ann. of Math., 137 (1993), 129-144.

[Zh9] Homotopy of projections and factorization in the unitary groups of certain $C^{*}$-algebras, Math. Scand., 73 (1993), 281-306.

[Zh10] On the exponential rank and exponential length of $C^{*}$-algebras, J. Operator Theory, 28 (1992), 337-355.

[Zh11] On the homotopy type of the unitary group and the Grassmann space of purely infinite simple $C^{*}$-algebras, K-Theory, to appear.

Received April 8, 1994 and revised October 15, 1996. The author was partially supported by NSF and a Taft travel grant.

UNIVERSITY OF CinCINNATI

Cincinnati, OH 45221-0025

E-mail address: szhang@ucbeh.san.uc.edu 